\title{
Morphological appearances of a bacterial L-form growing in association with the erythrocytes of arthritic subjects
}

\author{
PHYLLIS PEASE \\ From the Department of Bacteriology, University of Birmingham
}

In a previous paper (Pease, 1969), it was shown that the L-form of a Gram-positive bacterium, provisionally identified as a species of Listeria, can be isolated by simple methods from the blood and synovial fluid of arthritic subjects, in which they are more numerous than in normal persons (Pease, 1967).

Further work has shown that these L-forms grow, not only as simple, budding spheroplasts (previously figured, Pease, 1969), but also in association with the erythrocytes, where they reveal a range of morphologies similar to the classical pleuropneumonia organisms, described by Turner (1935), Freundt (1952), and Klieneberger-Nobel and Cuckow (1935).

This paper is intended to provide a description of this phenomenon, which may be of significance, in view of the relationship between arthritis and haemolytic anaemias (Dacie, 1967), and the increased blood sedimentation rates also found in the disease.

\section{Methods}

Observations were made on whole blood or serum separated naturally from the clot, taken with aseptic precautions from 76 cases of rheumatoid arthritis in the St John's and Highfield Hospitals, Droitwich, and the Queen Elizabeth Hospital, Birmingham. The cases were diagnosed by clinical findings, $x$ rays, and serological tests, and specimens were provided by courtesy of Drs. C. F. Hawkins, A. J. Popert, and J. D. Goode. For comparison, a strain of Mycoplasma arthritidis (Campo) was examined; this was provided by courtesy of Dr. D. G. ff. Edward.

Primary cultures were made by one of two methods:

(1) $15 \mathrm{ml}$. of Brewer's medium (Oxoid) was placed in screw-stoppered McCartney bottles, and autoclaved for $15 \mathrm{~min}$. at $15 \mathrm{lb}$.; $2 \mathrm{ml}$. of the patient's serum was then added to each with sterile precautions. Incubation was at $37^{\circ} \mathrm{C}$.

(2) $10 \mathrm{ml}$. of a medium containing 1 per cent. peptone, 1 per cent. Lab. Lemco, $0 \cdot 25$ per cent. yeast extract, and 0.5 per cent. sodium chloride, in water at $\mathrm{pH} 7.8$ was placed in screw-stoppered McCartney bottles, and autoclaved for $15 \mathrm{~min}$. at $15 \mathrm{lb}$; $1 \mathrm{ml}$. of the patient's whole blood was then added to each with sterile precautions. Incubation was at $37^{\circ} \mathrm{C}$.

No native protein or other unsterilized material, other than the inoculum, was added to the medium, in either method, to avoid the possibility of contamination with foreign L-forms or mycoplasmas, against which filtration is not an adequate defence (Pease, 1967).

As a control against contamination, subcultures were made when the cultures were prepared, and at intervals thereafter (see Results) on PPLO agar (Difco), horse blood agar, heated horse blood agar, 10 per cent. glycerol agar (Oxoid base 2), Dorset's and Lowenstein-Jensen egg media; these were incubated at $37^{\circ} \mathrm{C}$.

Development of the growth on erythrocytes was observed in microcultures, viewed directly with a Wild $x$ 50 phase-contrast lens and condenser. Microcultures were prepared from the fluid cultures containing whole blood. After incubation of the primary culture for 3 days, a few drops of culture were placed on a sterile slide, under a sterile cover-slip, and sealed at the edges with sterile beeswax. These microcultures were incubated for $12 \mathrm{hrs}$, at the relatively low temperature of $25^{\circ} \mathrm{C}$., according to the technique originally employed by Turner (1935) for demonstrating the organism of bovine pleuropneumonia.

Positive results were achieved in approximately 90 per cent. of tests, after 3 days' incubation of primary cultures, followed by 12 hours' incubation of microcultures, but in the remaining cases, the primary cultures were positive after a further week, or, in one example, after 3 weeks.

Preparations for electron microscopy were made from the microcultures, by harvesting the contents of several with a Pasteur pipette, suspending in distilled water, permitting the erythrocytes to sediment naturally, and re-suspending twice; the sediment was then placed directly upon a membrane. These preparations were shadowed with gold palladium mixture.

\section{Results}

The primary cultures inoculated with serum were intended to confirm, with larger numbers, the observations previously reported in this journal (Pease, 1969), and they gave the same results. Visible turbidity, consisting of budding spheroplasts, appeared in all cultures within 2 to 6 weeks, usually in 
the shorter time, and the spheroplasts were readily seen in wet smears of centrifuged deposit, under the phase-contrast microscope (see Pease, 1969 for method and illustrations). In younger cultures, before development of visible turbidity, small numbers of spheroplasts could be seen, but it was not easy to distinguish them from debris, platelets, etc., until their concentration had increased.

In primary cultures inoculated with blood, and containing numerous erythrocytes, development of the L-form was much more rapid, and it was readily observed in the microcultures made from such primary cultures. As seen by phase-contrast (Figs 1 to 10), they exactly paralleled the appearances in the dark-ground photomicrographs of Turner (1935), illustrating the organism of bovine pleuropneumonia. In the present instance, further detail was provided also by the electron microscope (Figs 11 to 14). In the later stages of culture (Figs 6, 9, 10), the L-forms became free of the erythrocytes and grew in the medium, as in the case of the primary serum cultures.

In microcultures made from primary cultures that had been incubated for 3 days at $37^{\circ} \mathrm{C}$., themselves incubated for 12 to $24 \mathrm{hrs}$ at $25^{\circ} \mathrm{C}$., the erythrocytes showed numerous bulbs, varying from 0.3 to $1 \cdot 0 \mu$, attached by short stalks to the periphery of the

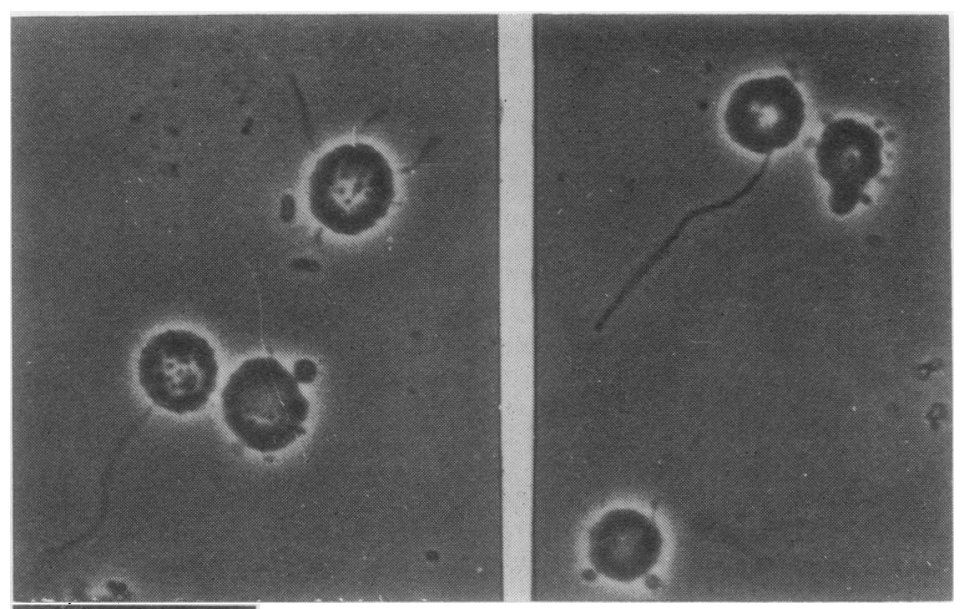

FIGS 1, 2 Erythrocytes in microculture, showing bulbs and filaments. Phase-contrast. $\times 2,500$.

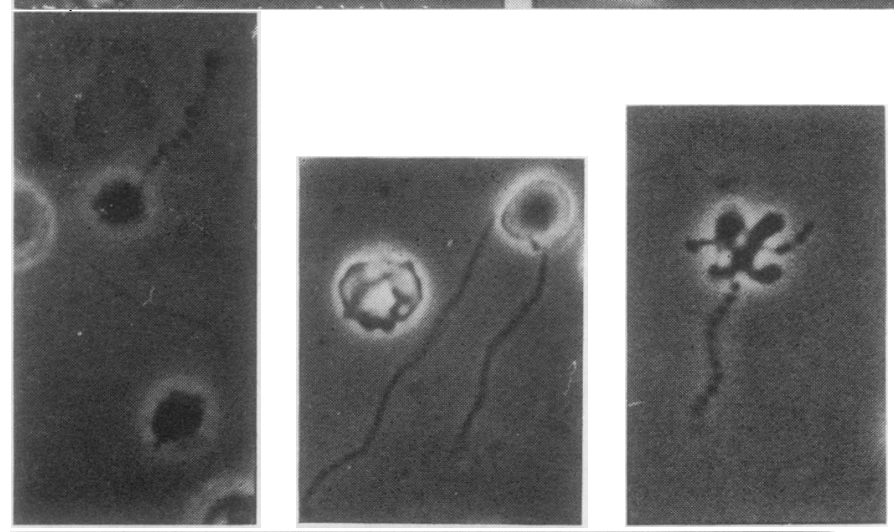

FIGS 3, 4, 5 Erythrocytes in microculture, showing filaments breaking down into chains of spherical bodies. Phase-contrast. $\times 2,500$.

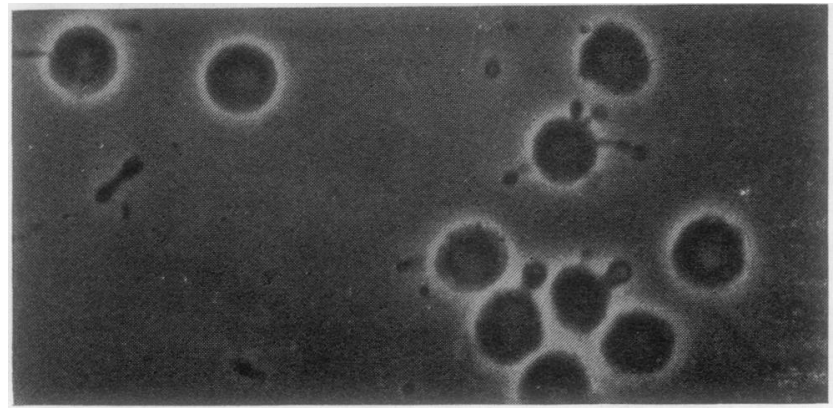

FIG. 6 Erythrocytes in microculture, showng stalked spheres and a single dumb-bell shaped rod. Phase-contrast. $\times 2,500$. 
(9)

(8)

(7)
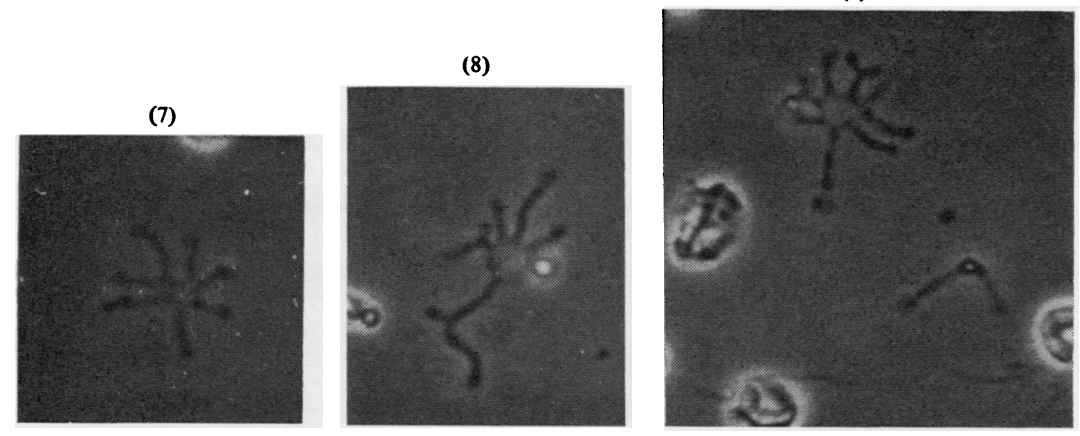

FIG. 7 Asterococcus form. Phase-contrast. $\times 2,500$.

FIGS 8, 9 Asterococcus forms with filaments commencing to ramify. Phasecontrast. $\times 2,500$.

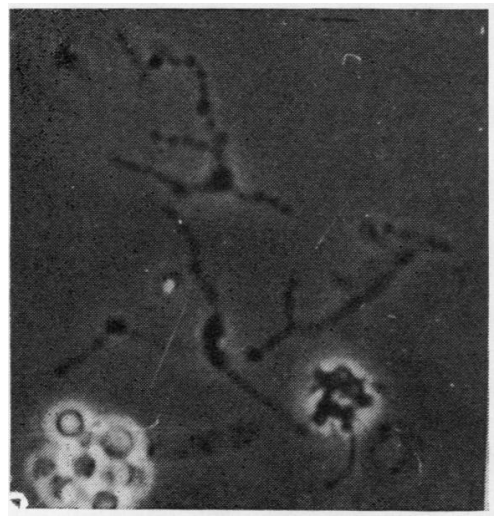

erythrocytes and slender filaments, c. $0 \cdot 1 \mu$ in width and up to 10 to $20 \mu$ in length, or even longer upon occasion (Figs 1, 2, 3). Some filaments could be observed in the process of transforming into chains of spherical bodies, superficially resembling streptococci (Figs 3, 4, 5). If they were further incubated and examined during this process, the number of chains increased in proportion, and the transformation could, upon occasion, be observed as it occurred under the microscope. Turner (1935) observed an identical process which he called genethode $I$. Similar filaments occur in cultures of mycoplasmas associated with arthritis in lower animals (compare Figs 12, 13, and 14 with Fig. 15).

A variant of this appearance, called genethode III by Turner, was frequently seen in early microcultures (Fig. 6). This consisted of rather larger spherical bodies, sometimes more than one on the same slender stalk, and superficially resembling the little-known sessile bacteria Hyphomicrobium. In addition to Turner, who worked with pure cultures, these bodies have been observed on erthrocytes by Tedeschi, Amici, Murri, and Paparelli (1967), who showed that the small, stalked bulbs developed from granular bodies with the cell (Fig. 17), and by Villequez (1965), who described both the filaments and the hyphomicrobial forms.

The most characteristic mycoplasma appearances were found in microcultures prepared from older cultures ( 7 to 8 weeks). These were the 'asterococci' from which one of the former names of the pleuropneumonia organisms was derived (Figs 7, 8, 9). It is generally agreed (Turner, 1935; Freundt, 1952; Klieneberger-Nobel and Cuckow, 1955) that these develop from ring-shaped bodies (Figs 11, 14) by the growth of arm-like projections (Figs 7, 8, 9, 12, 14). The electron micrographs may be compared with the appearances in mycoplasmas associated with animal arthritides (Fig. 16), and both electron and phase-contrast micrographs with the dark-ground photomicrographs of Turner, and the electron micrographs of Freundt and of Klieneberger-Nobel and Cuckow. The sprouting arms then ramify to produce a small mycelium that then breaks down into coccal forms, in the same manner as the simple filaments, previously described (Figs 8, 9, 10). This represents Turner's genethode II. Small clubbed and dumb-tell-shaped rods (Turner's genethode $V$ ) were also visible (Fig. 6). See also Ørskov (1942).

\section{CULTURAL CONTROLS}

To check that the cultures were pure at all times, and that the different morphological forms were stages in the development of a single micro-organism, subcultures on solid media were made from the original serum, at the time of inoculation of the primary cultures, and at random times during the 


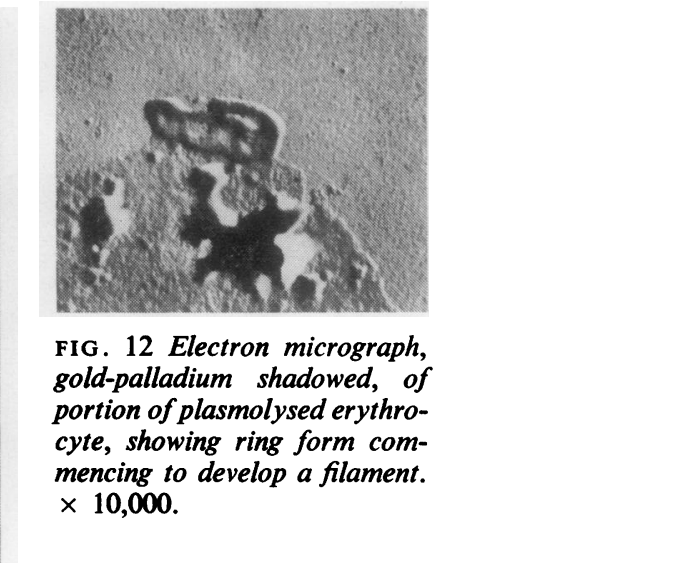

FIG. 11 Electron micrograph, gold palladium shadowed, of plasmolysed erythrocyte, showing ring forms attached to surface. $\times 10,000$

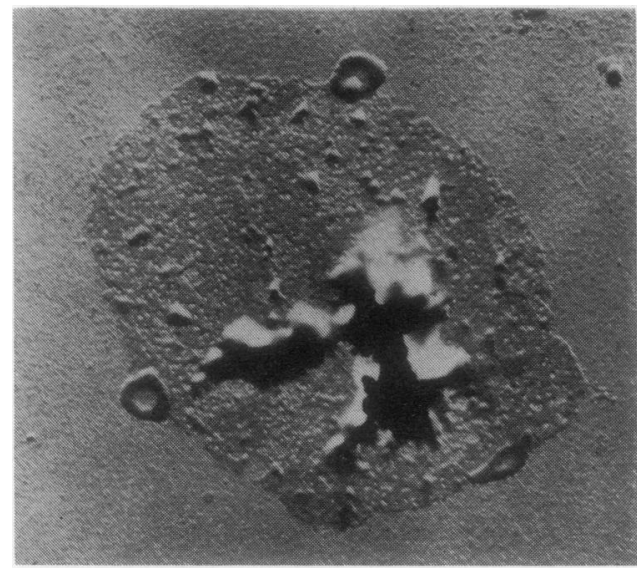

\section{forms atrached to surface. $\times 10,000$}
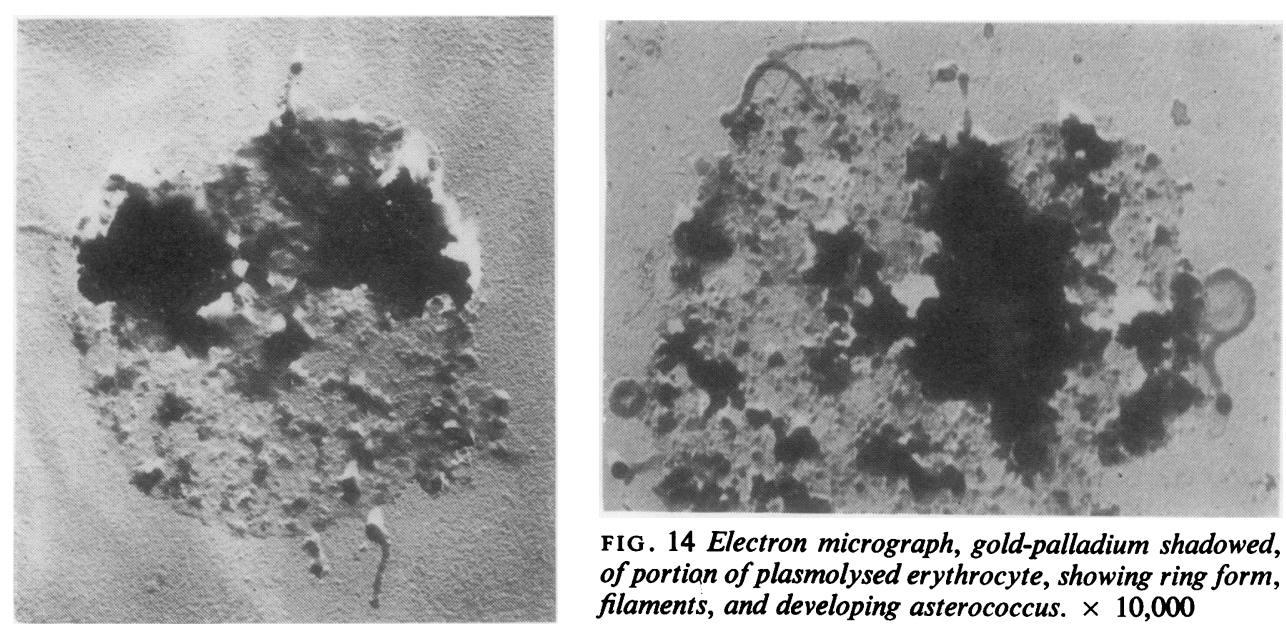

FIG. 14 Electron micrograph, gold-palladium shadowed, of portion of plasmolysed erythrocyte, showing ring form, filaments, and developing asterococcus. $\times 10,000$

FIG. 13 Electron micrograph, gold-palladium shadowed, of plasmolysed erythrocyte, showing attached filaments. $\times 10,000$

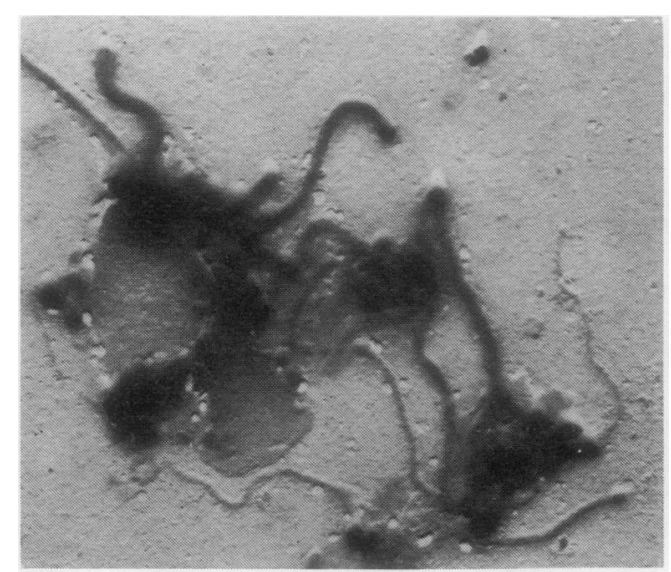

FIG. 15 Electron micrograph, gold-palladium shadowed. Culture of Mycoplasma arthritides, showing filaments. $\times 10,000$.

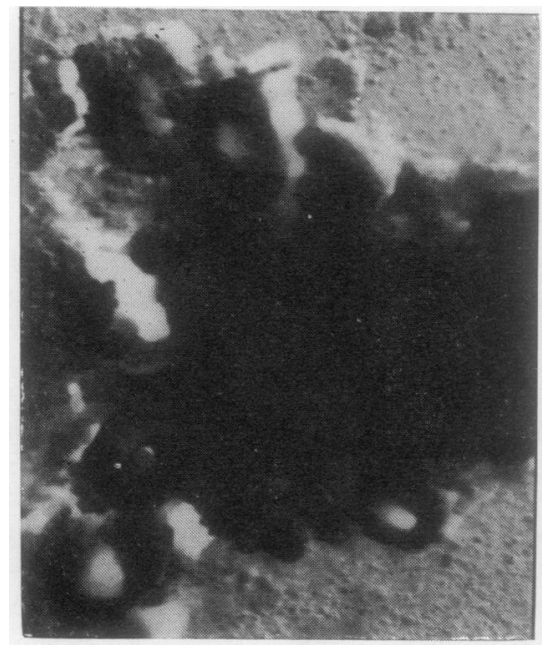

FIG. 16 Electron micrograph, gold-palladium shadowed. Ciuture of Mycoplasma arthritides, showing ring forms. $\times 20,000$. 


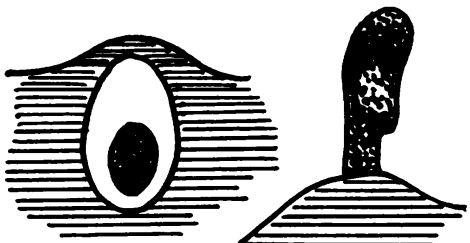

(a)

(b)

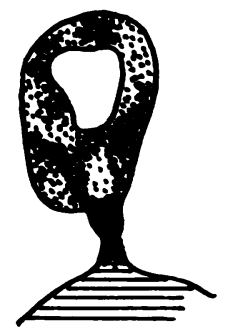

(c) process of incubation, on 20 per cent. of the primary cultures. One loopful of blood, serum, or culture medium was spread on the suiface of the media listed under Methods. These were dispensed in Petri dishes, except the egg media, which were sloped in screw-stoppered McCartney bottles. In the case of the blood cultures, the egg media were omitted from the controls; partly because egg contains numerous small granular bodies that made the growth of the L-form visually difficult to detect (Pease, 1967), and also because, in this case, it was the potential presence of a contaminating mycoplasma against which the controls were directed, and these are best tested on blood or heated blood agar or PPLO agar. Glycerol agar was $\epsilon$ mployed, in a later stage of the investigation, for the purpose of detecting and isolating the reverting bacillary forms of the L-form, that occurred in fluid primary cultures (Pease, 1967, 1969), although these grew also, if less well, on all the solid media described. Incubation was for one week at $37^{\circ} \mathrm{C}$. in all cases.

All these controls were completely clear after one week's incubation: that is to say, no growth was obtainable on any medium from the original blood or serum. From developing primary cultures, usually a mixed growth of L-forms and small bacillary forms was observable as a very thin film in the areas of inoculation. This was frequently so tenuous as to be overlooked by those not familiar with the type of work. L-forms did not grow on glycerol agar. No sign of contaminating bacteria or mycoplasmas could be observed, although standard mycoplasmas (e.g. Mycoplasma hominis type 1, and $M$. arthritidis Campo) grew well on the blood or heated blood agar and PPLO agar, under the same conditions, and produced typical colonies. It was therefore assumed that the cultures could be regarded as pure.

Subcultures of the bacillary phase grew well, and the study of this organism, and its further identification by cultural, physiological, and immunological methods is proceeding. Preliminary experiments (undertaken in collaboration with Dr. C. F. Hawkins) suggest that a relationship exists between degree of allergic reaction to this micro-organism and severity of rheumatoid arthritis, and it is hoped to present this evidence at a later date.
FIG. 17 Drawing after the electron micrographs of ultrasections, by Tedeschi and others (1967). (a) granule under surface of erythrocyte (hatched); (b) and (c) development of bulb on short stalk.

\section{Discussion}

The observations recorded in this paper serve to confirm my previous description of bacterial L-forms in the blood of arthritic subjects, in an increased number of cases, and also further to explain the irregular results achieved by different investigators, some of whom have described mycoplasmas and bacterial L-forms in this disease, whereas others have been unable to detect any infective agent. The L-form is not only slow and difficult to grow in culture, but its morphology is very variable, and when growing in association with blood cells, it may be impossible to isolate. The bacterium has been provisionally assigned to the Listeria group (Pease, 1967, 1969), and is thus related to Erysipelothrix, according to the criteria of Wilson and Miles (1964): an organism that has often been associated with arthritides of lower animals. Its L-form is now shown to pass through a morphological cycle closely resembling that of the classical pleuropneumonia organisms, which have also been associated with arthritides of lower animals.

The evidence that rheumatoid arthritis is accompanied, in many cases, by a massive infection of erythrocytes with bacterial L-forms, attached to the surface of the cell, suggests an additional factor that may be concerned in the phenomenon of differential sedimentation rates. Probably the cyst-like bodies seen in the original L-form cultures (Pease, 1969) were heavily infected blood cells.

A further point may be made in reference to the haemolytic anaemia 'so frequently present in active cases of rheumatoid arthritis' (Dacie, 1962). This leading authority indicated that the evidence pointed to the possibility of a foreign antigenic system in the erythrocytes, although it had not, at that time, been recognized.

Although I do not wish to be interpreted as suggesting that infection of the blood, or even of the joint fluids, with mycoplasma-like L-forms is necessarily the sole factor in infection-induced arthritis, further support is lent to this concept by the recent demonstration by Warren, Marmor, Liebes, and Hollins (1969), that a congenitally transmissible agent from human lesions can cause polyarthritis in mice: the organism described in this 
paper can almost certainly be congenitally acquired (Pease, 1967). This type of infection seems to be acute in cases of arthritis, but it is found to some extent in many apparently normal persons. The disease must still be regarded as an immune disturbance, although it may be mediated by infection with sub-cellular bacterial elements, according to the types of mechanism suggested by me in a previous monograph (Pease, 1965).

Two obvious and much-quoted potential sources of error must be discussed at this point. Configurations claimed to resemble the forms illustrated here as typical of classical pleuropneumonia organisms have been described frequently as 'myelin formations', and are supposed to be caused by droplets of free lipid, especially cholesterol and its derivatives, in an aqueous medium (Wilson and Miles, 1964). It is, however, apparent that this confusion has extended to genuine mycoplasma cultures (Partridge and Klieneberger, 1941; Villequez, 1965), probably becausz these microorganisms actually have a high lipid content. In the present case, many bizarre forms were observed and disregarded; usually these were many times larger than the structures illustrated as mycoplasmalike L-forms in this paper, and resembled the accidental lecithin formations illustrated by Partridge and Klieneberger (1941). The mycoplasma forms could not only be observed to grow in microcultures, under the microscope, but retained substantially the same morphology in the electron micrographs of erythrocytes after plasmolysis by distilled water. The earlier, granular stages were identical with those shown in the erythrocyte, in electron micro- graphs of ultra-sections, by Tedeschi and others (1967) (Fig. 22). Tedeschi, Amici, and Paparelli (1969) obtained evidence of nucleoside metabolism in erythrocytes, that they attributed to parasitism by $\mathrm{L}$-forms. The same cycle was followed in culture by Turner (1935) and Ørskov (1942), and resemblances between the forms described and figured by different authors, using different methods, are much too close to be accidental. The second potential source of error is obviously contamination, and it is apparent that the possibility can never be absolutely eliminated. It can only be stated here that rigorous controls were used, and that within the limits of these methods, the purity of the cultures was assured.

\section{Summary}

Evidence is presented indicating the close association of what is believed to be a bacterial L-form with the erythrocytes of arthritic subjects. Both phase-contrasts and electron micrographs showed very strong resemblances between these $\mathrm{L}$-forms and classical pleuropneumonia-like organisms. The close similarity between these and mycoplasmas found in association with arthritis in lower animals is pointed out.

I wish to acknowledge the help and encouragement of Dr. K. A. Bisset, the valuable assistance of Drs. C. F. Hawkins, A. J. Popert, and J. D. Goode, and the skilled technical work of Mr. C. C. Newton, Mrs. C. M. F. McCaughey, and Miss Judy Johnston. I also wish to thank the Arthritis and Rheumatism Council for their provision of a phase-contrast microscope, and for assistance in respect of animals for serological work.

\section{References}

Dacie, J. V. (1962) 'The Haemolytic Anaemias', 2nd ed., Part 2, p. 604. Churchill, London.

- (1967) Idem, Part 3, p. 823.

Freundt, E. A. (1952) Acta path. microbiol. scand., 31, 508 (Morphological studies of the peripneumonia organism, Micromyces peripneumonia bovis).

KLIENEBERGER-Nobel, E., AND CuCKOW, F. W. (1955) J. gen. Microbiol., 12, 95 (A study of organisms of the pleuropneumonia group by electron microscopy).

ØRSKOV, J. (1942) Acta path. microbiol. scand., 19, 586 (On the morphology of peripneumonia-virus, agalactiavirus and Seiffert's microbes).

Partridge, S. M., ANd Klieneberger, E. (1941) J. Path. Bact., 52, 219 (Isolation of cholesterol from the oily droplets found in association with the L1 organism separated from Streptobacillus moniliformis).

Pease, P. E. (1965) 'L-forms, episomes and auto-immune disease'. Livingstone, Edinburgh.

- (1967) Nature (Lond.), 215, 936 (Tolerated infection with the sub-bacterial phase of Listeria). (1969) Ann. rheum. Dis., 28, 270 (Bacterial L-forms in the blood and joint fluids of arthritic subjects).

Tedeschi, G. G., Amici, D., Murri, O., ANd PaParelli, M. (1967) Haematologica, 52,57 (Aspetti in microscopia ottica ed elettronica del sangue di pazienti splenectomizzati o affetti da ittero emolitico).

,,-- AND PaPARelli, M. (1969) Nature (Lond.), 212, 1285 (Incorporation of nucleosides and amino acids in human erythrocyte suspensions: possible relation with a diffuse infection of mycoplasms or bacteria in the $L$ form).

TURner, A. W. (1935) J. Path. Bact., 41, 1 (A study of the morphology and life cycles of the organism of Pleuropneumonia contagiosa boum (Borrelomyces peripneumoniae nov. gen.) by observation in the living state under dark-ground illumination).

VilleQuez, E. (1965) Gaz. méd. Fr., 72, 535 (Le parasitisme latent du sang).

WARren, S. L., MARmor, L., Liebes, D. M., AND Hollins, R. L. (1969) Nature (Lond.), 223, 646 (Congenital transmission in mice of an active agent from human rheumatoid arthritis).

Wilson, G. S., AND Miles, A. A. (1964) “Topley and Wilson's Principles of Bacteriology and Immunity", Sth ed., pp. 532 and 1150. Arnold, London. 